\title{
Advances in Smartphone-Based Point-of-Care Diagnostics
}

\author{
This paper reviews the state-of-the-art advances in smartphone-based point-of-care \\ diagnostic technologies and their applications in medicine and biology. \\ By Xiayu Xu, Altug Akay, Huilin Wei, ShuQi Wang, Belinda Pingguan-Murphy, \\ Björn-Erik Erlandsson, Xiujun Li, WonGu Lee, Jie Hu, Lin Wang, and Feng Xu
}

\begin{abstract}
Point-of-care (POC) diagnostics is playing an increasingly important role in public health, environmental monitoring, and food safety analysis. Smartphones, alone or in conjunction with add-on devices, have shown great capability of data collection, analysis, display, and transmission, making them popular in POC diagnostics. In this article, the state-ofthe-art advances in smartphone-based POC diagnostic technologies and their applications in the past few years are outlined, ranging from in vivo tests that use smartphone's built-in/external sensors to detect biological signals to in vitro tests that involves complicated biochemical reactions. Novel
\end{abstract}

Manuscript received July 1, 2014; revised October 29, 2014; accepted November 21, 2014. Date of current version March 23, 2015. This work was financially supported by the National 111 Project of China (B06024), the Key (Key grant) Project of Chinese Ministry of Education (313045), South Wisdom Valley Innovative Research Team Program, International Science \& Technology Cooperation Program of China (2013DFG02930), Key Program for Intemational S\&T Cooperation Projects of Shaanx (2014 KW12-01), the Fundamental Research Funds for the Central Universities, National Natural Science Foundation of China ( 81401480 ), and China Postdoctoral Science Natural Science Foundation of China (81401480), and China Postdoctoral Science Foundation (2013M532054, 2014M552460). B.P-M. received funding from the Ministry grant (UM.C/HIR/MOHE/ENG/44).

grant (UM.C/HIR/MOHE/ENG/44).
X. Xu, J. Hu, L. Wang, and F. Xu are with the MOE Key Laboratory of Biomedical $X . X u, J . H u, L$ Wang, and F. Xu are With the MoE Key Laboratory of Biomedical Information Engineering, School of Life Science and Technology, X'an fiaoton University, XI' an, China. They are also with the Bioinspired Engineering and Biomechanics Center (BEBC), Xi'an Jiaotong University, Xi'an, Chin (e-mail: wanglin0527@126.com; changie.hu@gmail.com).

A. Akay and B.-E. Erlandsson are with the School of Technology and Health, Royal Institute of Technology, Huddinge, SE-141 52 Sweden.

H. Wei is with the Novatein Biosciences, Inc., Woburn, MA, 01801 USA. S. Wang is with the MOE Key Laboratory of Biomedical Information Engineering, School of Life Science and Technology, Xi'an Jiaotong University, Xi'an, China. He is also with the Bio-Acoustic MEMS in Medicine (BAMM) Laboratory, Canary Center at Stanford for Cancer Early Detection, Department of Radiology, Stanford School of Medicine, Palo Alto, CA 94304 USA. He is also with the State Key Laboratory for Diagnosis an Treatment of Infectious Diseases, First Affiliated Hospital, College of Medicine, Zhejiang University, Hangzhou, China, and with the Collaborative Innovation Center for Diagnosis and Treatment of Infectious Diseases, Hangzhou, China, and also with the Institute for Translational Medicine, Zhejiang University, Hangzhou, China.

B. Pinguan-Murphy is with the Department of Biomedical Engineering. Faculty of Engineering, University of Malaya, Kuala Lumpur 50603, Malaysia.

$X$. Li is with the Border Biomedical Researh Center, Biomedical Engineering (BME) and x. Li is with the Border Biomedical Research Center, Biomedical Engineering (BME)

TX 79902 us

W. Lee is with the Department of Mechanical Engineering, Kyung Hee University, Korea.

Digital Object Identifier: 10.1109/JPROC.2014.2378776

18-9219 (C) 2015 IEEE. Personal use is permitted, but republication/redistribution requires IEEE permission.
See http://wwww.ieee.org/publications_standards/publications/rights/index.html for more information.

236

Proceedings of the IEEE | Vol. 103, No. 2, February 2015 techniques are illustrated by a number of attractive examples, followed by a brief discussion of the smartphone's role in telemedicine. The challenges and perspectives of smartphonebased POC diagnostics are also provided.

KEYWORDS | Mobile medicine; point-of-care (POC) diagnostics; public health; smartphone

\section{INTRODUCTION}

As a form of test performed at or near the test site, point-ofcare (POC) diagnostics has received increasing attention in recent years [1]-[9]. POC diagnostics offers several advantages compared with laboratory-based tests in that the former is normally portable, inexpensive, rapid, and easyto-use [10]. These features have provided POC diagnostics with an indispensable role in global and public health, such as in the control and treatment of infectious and chronic diseases [11]-[13]. For example, it can provide timely diagnostics for tuberculosis (TB) and human immunodeficiency virus (HIV), effectively preventing the spread of these diseases, and provide continuous, long-term monitoring services for diabetes mellitus and cardiovascular diseases [14]-[17]. Besides, POC diagnostics has shown great potential in environmental monitoring and food safety analysis [18], [19]. Therefore, the development of POC diagnostic technologies becomes increasingly urgent.

The three phases of a POC test are preanalytical, analytical, and postanalytical [20]. Preanalytical phase includes selection of proper test methods and specimen collection. Analytical phase is the process of detecting targeted biological signals and transforming them into measurable signals. Postanalytical phase includes data analysis, result display, storage and transmission, and decision-making. Early POC technologies usually require extra peripheral devices for analytical and postanalytical evaluation (e.g., electronic sphygmomanometer), thus significantly increasing the cost and complexity in performance and limiting 
Table 1 Categories of Smartphone-Based Diagnostics

\begin{tabular}{|l|l|l|l|}
\hline Category & Explanation & \multicolumn{2}{|l|}{ Examples } \\
\hline $\begin{array}{l}\text { In vivo } \\
\text { test }\end{array}$ & $\begin{array}{l}\text { Tests that do not require } \\
\text { sample consumption; } \\
\text { biological signals are } \\
\text { converted to electrical signals } \\
\text { by various sensors. }\end{array}$ & $\begin{array}{l}\text { Test with } \\
\text { built-in sensor }\end{array}$ & $\begin{array}{l}\text { Use the built-in sensors, such as the camera, } \\
\text { to collect human body or environmental } \\
\text { signals. }\end{array}$ \\
\cline { 3 - 5 } & $\begin{array}{l}\text { Test with extra } \\
\text { sensor }\end{array}$ & $\begin{array}{l}\text { Use extra sensors, such as an ultrasound } \\
\text { probe, to collect human body or } \\
\text { environmental signals. }\end{array}$ \\
\hline \multirow{2}{*}{$\begin{array}{l}\text { In vitro } \\
\text { test }\end{array}$} & $\begin{array}{l}\text { Tests that require sample } \\
\text { consumption; } \\
\text { biological components or } \\
\text { organisms are detected from } \\
\text { samples, such as blood, sweat, } \\
\text { etc. }\end{array}$ & $\begin{array}{l}\text { Tube, strip, } \\
\text { and specimen } \\
\text { inspection }\end{array}$ & $\begin{array}{l}\text { Take a specimen of bodily fluid and directly } \\
\text { inspect the result using the built-in camera } \\
\text { ter a microscope connected to a smartphone. }\end{array}$ \\
\cline { 3 - 5 } & \multicolumn{2}{|l}{$\begin{array}{l}\text { Take a specimen of bodily fluid and use } \\
\text { microfluidic technique to perform } \\
\text { complicated biochemical tests, and visualize } \\
\text { the result using a smartphone. }\end{array}$} \\
\hline
\end{tabular}

their widespread applications in global and public health. Developing cost-effective and easy-to-operate POC technologies is therefore desirable.

Recent advances in smartphone technologies hold great potential to solve these problems. Smartphones, equipped with a computer-like platform and various types of sensors, have several properties promoting their uses in POC diagnostics [21]. The global market has witnessed a rapid growth of smartphones in recent years. Reports from International Data Corporation (IDC) and Canalys state that the number of smartphone subscriptions worldwide has reached up to 1.0 billion in 2013, and the number is expected to surpass 1.2 billion in 2014, driven by rapid growth in developing countries (e.g., India and China) [22], [23]. This means that smartphones are becoming widely accessible even in resource-limited areas lacking adequate healthcare facilities. Furthermore, a rich set of built-in sensors (e.g., camera and microphone) can be used for the detection of biological signals, powerful processors and memories for the analysis and storage of diagnostic results, and high resolution screens for result display [24], [25]. Finally, smartphones are generally equipped with powerful data transmission capabilities, such as Global System for Mobile Communication (GSM), wireless fidelity (Wi-Fi), Bluetooth, and universal serial bus (USB), allowing short-distance and long-distance communication between a remote test site and centralized laboratory for professional guidance.

Over the past few years, there has been a significant increase in smartphone-based healthcare technologies, as reflected by over 40,000 mobile health applications available in 2012 [26]. A number of articles have reviewed these advances: Patrick et al. and Wang et al. reviewed the application of smartphone in healthcare respectively in 2008 and 2009 [27], [28]. Xie et al. reviewed the development of biomedical imaging techniques combined with smartphones in 2010 [29]. With the rapid development of smartphone, many novel features are available now, and many new healthcare technologies have been introduced. A more recent review by Agu et al. focused on the usage of smartphone in medical condition diagnostics that takes advantage of the smartphone's built-in camera or microphone [30]. Another recent review by Ozcan et al. focused on the uses of smartphone for imaging/microscopy and optoelectronic/electronic sensing, such as smartphonebased microscopy that can detect single virus, as well as smartphone-based cytometry [31]. These existing reviews have not focused on the combination of smartphone and POC diagnostics or only focused on a single area of smartphone-based POC diagnostics. Here, we review the latest developments in smartphone-based POC diagnostics, ranging from in vivo tests that use smartphone's built-in/ external sensors to detect biological signals to in vitro tests that are combined with complicated biochemical reactions (Table 1). Novel techniques are introduced and illustrated by a number of attractive examples, followed by a brief discussion of the smartphone's role in telemedicine. Last, we present the challenges and perspectives in smartphone-based POC diagnostics.

\section{IN VIVO TESTING}

In vivo tests capture health information from the target without sample consumption. Some biological signals, such as two-dimensional (2D) color images and sounds, can be directly captured using a smartphone. Furthermore, more sophisticated diagnostic information can be obtained by connecting smartphone with add-on devices [32], [33].

\section{A. Smartphone-Based POC Diagnostics With Built-in Sensors}

Although a variety of sensors have been imbedded in smartphone, the widely used sensors in POC diagnostics are still limited to camera and microphone. A large amount 


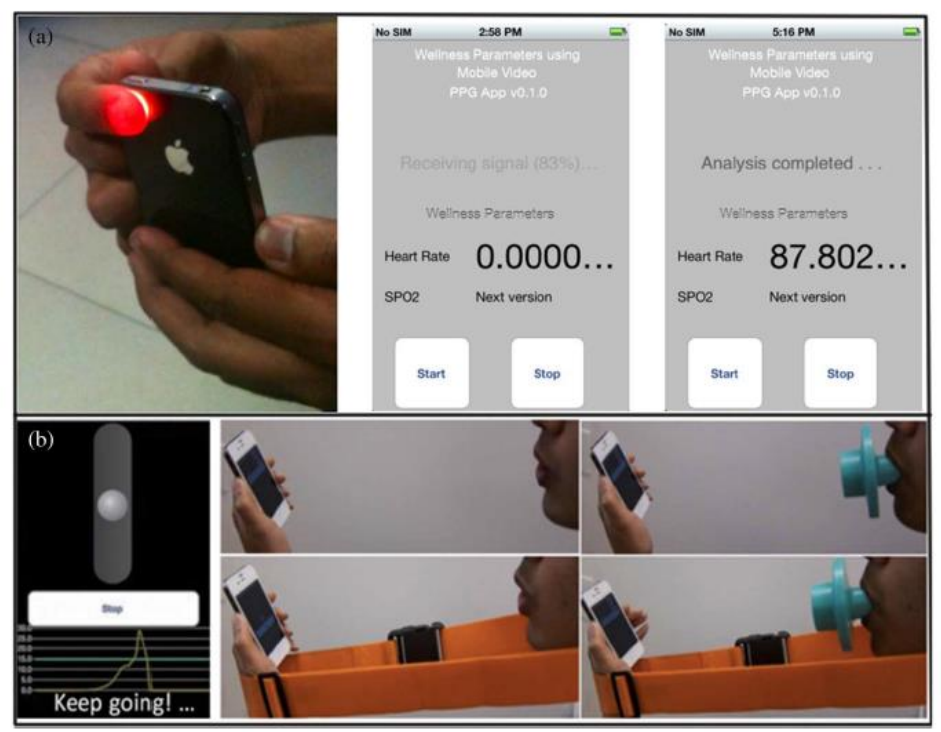

Fig. 1. Examples of in vivo POC diagnostics with smartphone's built-in sensors. (a) Heart rate detection from a fingertip [35]. (b) POC spirometer by recording the sound of exhalation using smartphone's built-in microphone [41].

of diagnostic information can be extracted from the raw audio or video data when combined with signal or image processing algorithms.

The megapixel count of the smartphone's built-in camera has been doubled in every two years in recent decades and is now as high as 41 megapixels (Nokia 808 PureView). Researchers are able to extract various types of health information from images of the human body, such as fingertips, eyes, and skin using image and/or video postprocessing techniques performed either in a smartphone or computer. Widely used image processing algorithms include Fourier transform [34], [35], color signal analysis [36], region segmentation [37], and pattern recognition [38], [39]. For example, fingertips contain abundant information about blood circulation. Jonathan et al. [34] and Pal et al. [35] obtained changes in heart rate by capturing photoplethysmographic (PPG) signals from a fingertip using a reflection PPG imaging technique [Fig. 1(a)]. To collect PPG signals from a fingertip, a smartphone was used to detect, record, and process the reemitted signals of a white light emitting diode (LED) illumination source. Similarly, Scully et al. achieved monitoring of various physiological signals, including cardiac R-R intervals, breathing rate, and blood oxygen saturation [36].

In addition to obtaining blood circulation status from fingertips, a bunch of other smartphone-based technologies have been developed. For example, a simple smartphonebased pupilloeter was developed to measure the diameter of pupil, providing information on the function of autonomic nervous system [37]. By comparing tongue images acquired using a smartphone with an image database, Samsung Electronics Company developed a method to determine the overall health status of a person (e.g., fatigue status) [38]. Similarly, Wadhawan et al. developed a smartphone-based melanoma detection technology [39].

Audio information taken by the smartphone's built-in microphone, combined with digital signal processing algorithms, is also used to acquire health information. Yoshimine et al. reported the use of a voice-recording function to diagnose the overall health status of individuals by comparing to the voice database from healthy individuals [40]. Larson et al. reported a smartphone-based spirometer, in which the sound of exhalation is recorded and analyzed for lung function [see Fig. 1(b)] [41]. Thus, smartphone-based POC technologies have been rapidly developed to collect and monitor basic health information in nonclinical settings.

\section{B. Smartphone-Based POC Diagnostics With}

External Sensors

So far, the information extracted by smartphone's builtin sensors is mainly limited to images and sounds. Many external sensor systems have been designed and integrated into the smartphone to extend its capability to extract more sophisticated diagnostic information, such as body temperature and functional images of organs and tissues. This allows previously unattainable health information to be extracted using external sensors and processed or 


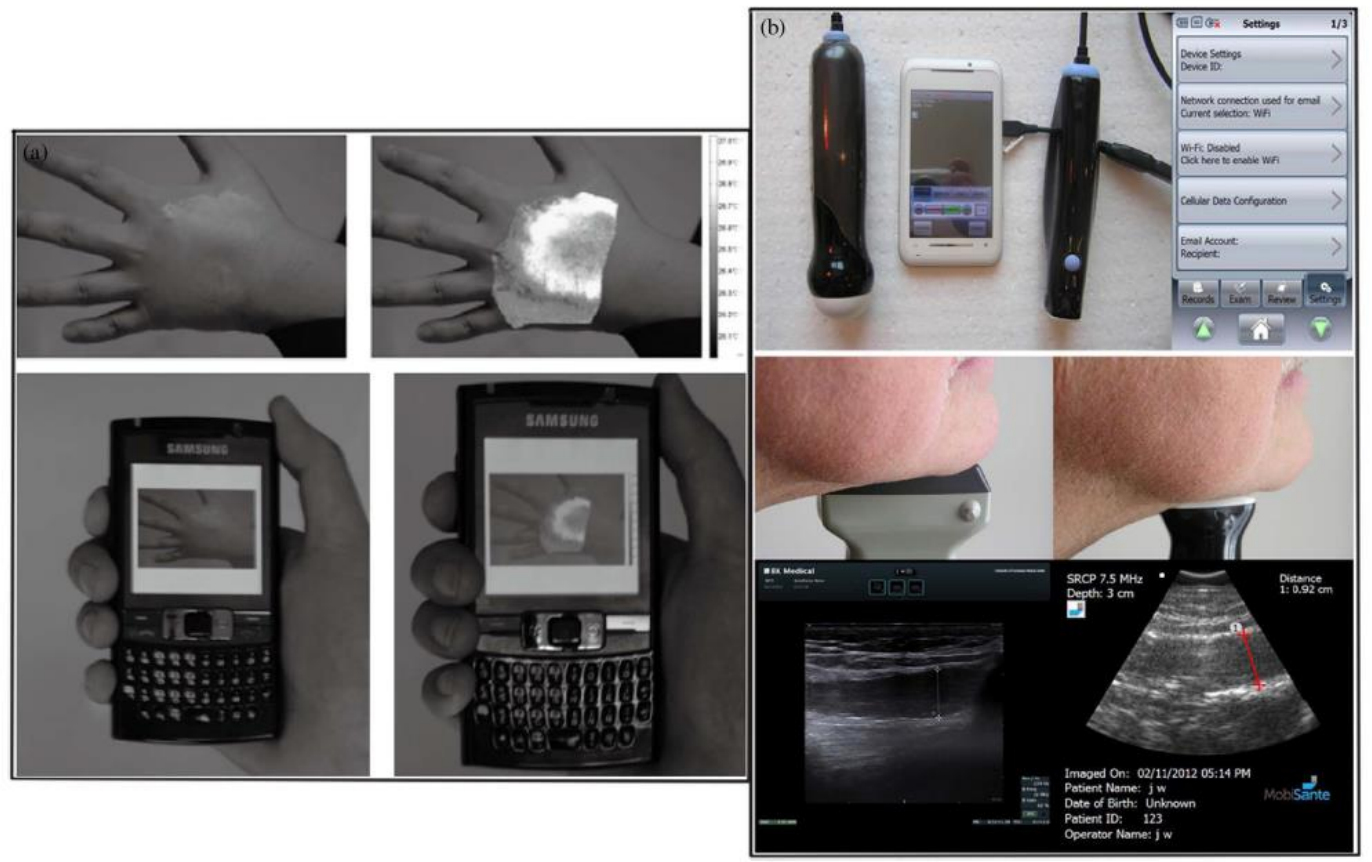

Fig. 2. Examples of in vivo POC diagnostics with extra sensors. (a) Skin temperature detection by mapping skin temperature dhanges to TLC color changes [42]. (b) POC ultrasound imaging system (Mobisante Mobius SP1 system) with two mechanical sector uSB probes [44].

transmitted using a smartphone in the form of onedimensional (e.g., body temperature and pulse rate), twodimensional (e.g., ultrasound image), or three-dimensional (e.g., time-sequence ultrasound images) signals.

Huang et al. developed a smartphone-based thermal imaging technology to quantitatively measure the temperature of human skin [42]. In this method, liquid crystal thermal (TLC), showing temperature changes in different color, was prepainted on human skin. The color changes were then captured as two-dimensional images using a smartphone's built-in camera and analyzed in a personal computer to measure the final temperature [see Fig. 2(a)] [42]. Khandoker et al. developed a smartphone-based lowcost oximeter photoplethysmo-graphy [43], in which the desired information, including blood oxygen saturation and pulse rate, was collected using a hardware system that can detect the absorption of red and infrared signals through a fingertip. The digital signals were then transmitted to a smartphone through USB for diagnostic result display and data communication between on-site patients and off-site clinicians.

Smartphone-based medical imaging is an important emerging area in POC diagnostics. Medical imaging, different from smartphone-based microscopy introduced in Section III-A, is the technique applied to create images of the human body (or function and parts) for clinical purposes, such as X-ray, computed tomography (CT), optical coherent tomography (OCT) and ultrasound. With the capability of providing high-resolution images of internal structure of human body, medical imaging has been widely used in the evaluation and diagnosis of many diseases. However, the high cost and need for highly trained skill to operate these clinical devices prohibit such imaging technologies from many remote regions. With significant advances in smartphone's display and processing capabilities, medical imaging combined with smartphone has become a research area with great potential [29]. MobiSante developed an ultrasound probe that is able to be plugged into a smartphone [Fig. 2(b)] [44]. With an ultrasonic transducer, the smartphone can acquire and display ultrasound images, which can then be transmitted to an off-site health center for further interpretation. Using this system, they obtained images of the suprahyoid airway and muscular architecture of mouth floor.

\section{IN VITRO TESTING}

In vitro tests are biochemical tests that detect/measure biological components (e.g., metabolites, proteins, and nuclei acids) and organisms (e.g., cells and microbes) from 


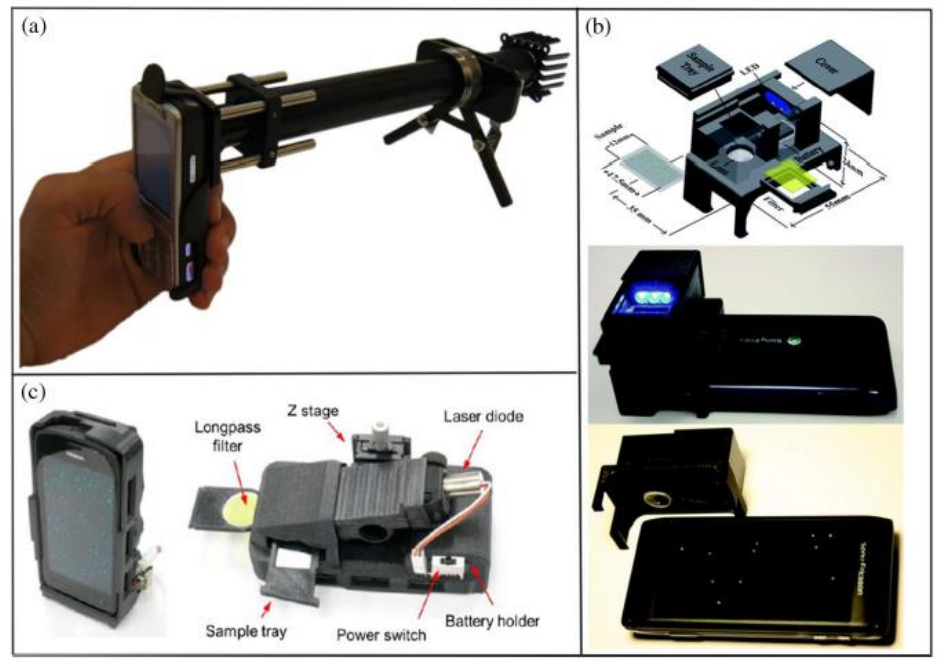

Fig. 3. Examples of smartphone-based microscopy systems. (a) Smartphone microscopy optical layout for fluorescence imaging [48]. (b) Schematic diagram and different views of the designed optical attachment for wide-field fluorescent imaging on a cell-phone [49]. (c) A cell phone-based fluorescence microscope [50].

blood, sweat, saliva, urine, water, or food [11], including conventional microscopy, widely used lateral flow assays, and lately developed microfluidic devices.

A. Recent Developments of Smartphone-Based Microscopy

Microscopy, which allows the microscale investigation of biological specimens, is widely used in biochemical tests to identify objects (e.g., cells, bacterium, and parasites) that cannot be visualized directly by naked eyes [45], [46]. Microscopes can be used in specimen tests, microfluidic tests, or any other form of in vitro tests that need visualization in microscale. Conventional lab microscopy is relatively costly, bulky, and requires a highly trained staff, impeding its application to near-patient diagnosis. In response, researchers have developed accurate, cost-effective, and easy-toperform microscopy, as a general tool suitable for POC applications using smartphones. Hence, before we delve into any specific smartphone-based biochemical diagnostic technique, we briefly review the recent development in smartphone-based microscopic imaging techniques.

Most smartphone-based microscopes are optical microscopes that consist of a visible light source and a system of lenses to magnify images of small objects. Image resolution and field-of-view (FOV) are two main parameters to evaluate the optical microscope's performance. Smith et al. developed a microscope attached to a smartphone that transformed the phone's integrated lens to a $350 \times$ microscope and visible-light spectrometer [47]. The microscope has a resolution of $1.5 \mu \mathrm{m}$ and a usable FOV of $150 \times$
$150 \mu \mathrm{m}$ without image processing and approximately $350 \times 350 \mu \mathrm{m}$ with postprocessing. Breslauer et al. reported a smartphone-mounted light microscope and obtained a resolution of $1.2 \mu \mathrm{m}$ and a usable FOV of $180 \times$ $180 \mu \mathrm{m}$ by adding a ball-lens to the system [see Fig. 3(a)] [48]. Zhu et al. demonstrated a wide-field fluorescent and dark-field imaging technique on a smartphone, in which a specimen was excited by a battery powered LED, after which the fluorescent emission from the sample was imaged using an additional lens positioned in front of the built-in camera [see Fig. 3(b)] [49]. This smartphone-based microscopy showed a large FOV of $\sim 81 \mathrm{~mm}^{2}$ with a raw spatial resolution of $\sim 10 \mu \mathrm{m}$. Wei et al. reported a fieldportable fluorescence microscopy platform installed on a smartphone with high spatial resolution that is able to image both individual nanoparticles $(100 \mathrm{~nm}$ of fluorescent particles) and viruses (fluorescently labeled human cytomegaloviruses) [see Fig. 3(c)] [50].

A type of lens-free microscope has been recently developed that obviates the need for any lenses or other optical components [51]. Tseng et al. reported a lens-free holographic microscope attached to a cellphone with a spatial resolution of $1.5 \sim 2 \mu \mathrm{m}$ over a FOV of $\sim 24 \mathrm{~mm}^{2}$ [52]. The additional hardware ( $\sim 38$ grams) installed on the cellphone is composed of an inexpensive LED (at $587 \mathrm{~nm}$ ) with an aperture of $\sim 100 \mathrm{~mm}$ in front of the light source.

The development in smartphone-based microscopy greatly strengthens and expands the capability of smartphone in POC diagnosis, especially in direct specimen examination. Microscale imaging opens an avenue for

Link to Full-Text Articles :

http://ieeexplore.ieee.org/xpls/abs all.jsp?arnumber $=70670228 t a g=1$ 